\title{
Effects of Catalyst on Gasochromic Properties in Tungsten Oxide Films
}

\author{
A. Inouye, S. Yamamoto*, S. Nagata, M. Yoshikawa*, T. Shikama \\ Institute for Materials Research Tohoku University, 2-1-1 Katahira Aoba-ku Sendai 980-8577 \\ Fax: 81-22-215-2062, e-mail: aichi@imr.tohoku.ac.jp \\ *Japan Atomic Energy Agency, 1233 Watanuki Takasaki 370-1292
}

Effects of catalyst on gasochromic properties in tungsten oxide films were investigated. Amorphous $\mathrm{WO}_{3}$ films coated with $\mathrm{Pd}$ and $\mathrm{Pt}$ catalysts were prepared on $\mathrm{SiO}_{2}$ substrates by employing reactive $\mathrm{RF}$ magnetron sputtering. To quantitatively estimate the thickness of the catalytic layer, the Rutherford backscattering spectroscopy (RBS) was employed. The coloration behavior was examined by measuring light transmittance of the samples exposed to $1 \%$ hydrogen diluted nitrogen gas. The field emission scanning electron microscope (FE-SEM) showed that particles with a dozes nm diameter of Pd and with a few $\mathrm{nm}$ of Pt grew on the surface of silicon substrates at beginning of deposition. The continuous catalytic layers were observed with increasing the amounts of more than $2 \mathrm{~nm}$ for Pd and $0.2 \mathrm{~nm}$ for Pt catalysts, respectively. When the continuous layers formed on $\mathrm{WO}_{3}$ surface, the excellent gasochromic properties were obtained. The results indicated that Pt catalysts were suitable for high sensitive hydrogen sensors consisting of gasochromic $\mathrm{WO}_{3}$ films.

Key wards: gasochromism, tungsten oxide, hydrogen sensor, catalytic layer

\section{INTRODUCTION}

The fast detection of hydrogen leaks with a volume below the lower explosive limit (LEL) of $4 \%$ in air is an important technology. A great number of solid-state hydrogen gas sensors have been demonstrated [1]. One of the hydrogen detection methods is a fiber optic sensing, which has possibility of remote sensing and lack of sparking in explosive environment. Tungsten trioxide $\left(\mathrm{WO}_{3}\right)$ films coated with thin catalyst layer such as Pd, Pt, and Ni can be useful in fiber optic sensing of hydrogen owing to coloration by incorporation of dissociated hydrogen into the films (gasochromism) [2, 3]. Gasochromic coloration is limited by dissociation of hydrogen molecules in catalysts and by diffusion of hydrogen atoms in the $\mathrm{WO}_{3}$ films [4]. In previously, many reports on gasochromic $\mathrm{WO}_{3}$ films have indicated that amorphous $\mathrm{WO}_{3}$ films prepared by evaporation and sputtering methods exhibit the excellent gasochromic coloration [4-7]. In addition, to improve the gasochromic coloration of tungsten oxide films, modifications of catalysts such as controlling the crystalline structure, size of particles, and surface area are necessary. However, there are few reports for effects of catalysts on gasochromic properties in amorphous $\mathrm{WO}_{3}$ films [4-7]. The gasochromic coloring levels [5] and speeds $[4,6]$ have been investigated in tungsten oxide films with various thicknesses of Pt catalytic layers. The results indicate that gasochromic coloration appears with thicker Pt layer than $2 \mathrm{~nm}$ by using optical interference method and quartz crystal oscillators. It is important to evaluate the catalytic layer thickness precisely for understanding the effects of the catalysts on the properties. On the other hand, ion-beam analysis such as Rutherford backscattering spectroscopy (RBS) is suitable to evaluate the small amounts of heavy atoms on surface. The RBS can recognize the Pd and Pt atoms of $1 \times 10^{14}$ atoms $/ \mathrm{cm}^{2}$ on surfaces, converting the amounts into approximately $0.02 \mathrm{~nm}$ in Pd and Pt bulk by using bulk atomic densities.

In this study, we investigate the effects of catalytic layers on gasochromic properties in amorphous $\mathrm{WO}_{3}$ films. The catalytic layers of $\mathrm{Pd}$ and $\mathrm{Pt}$ were deposited on the amorphous $\mathrm{WO}_{3}$ by employing RF magnetron sputtering. Amounts of the catalysts were precisely estimated by RBS using ${ }^{4} \mathrm{He}$ ions. Morphologies of the catalysts layers on silicon wafers were observed by field-emittion scanning electron microscopy (FE-SEM) since a few $\mathrm{nm}$ layers on the $\mathrm{WO}_{3}$ films are difficult to

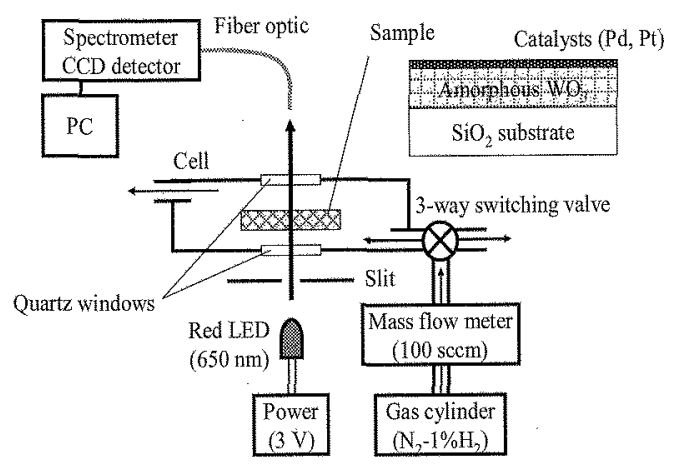

Fig.1 Experimental apparatus for examination of gosochromic coloration. 
be observed. To examine gasochromic properties of the $\mathrm{WO}_{3}$ films, changes of light transmittance were measured during samples were exposed to hydrogen gas. The optimization of catalysts thickness was also discussed for applications of the hydrogen sensors consisting of gasochromic $\mathrm{WO}_{3}$ films.

\section{EXPERIMENTAL}

Tungsten oxide films with Pd and Pt catalytic layers were prepared by employing the reactive RF magnetron sputtering method The tungsten oxides films were deposited by sputtering a $\mathrm{W}$ target (purity: $3 \mathrm{~N}$ ) in defined argon (purity: $6 \mathrm{~N}$ ) and oxygen (purity: $4 \mathrm{~N}$ ) mixture controlled by mass flow meters under the pumping condition. The films were deposited on $\mathrm{SiO}_{2}$ and mirror polished silicon substrates at a temperature of $200{ }^{\circ} \mathrm{C}$. The partial pressure of argon and oxygen were maintained at 150 and $20 \mathrm{mPa}$, respectively. The deposition rate corresponded to be $15 \mathrm{~nm} / \mathrm{min}$. In the deposition conditions, amorphous $\mathrm{WO}_{3}$ films were reported to be obtained in our previous study [8]. Catalytic Pd and Pt layers were deposited on the tungsten oxide films and silicon substrates by sputtering a $\mathrm{Pd}$ (purity: $3 \mathrm{~N}$ ) and $\mathrm{Pt}$ target (purity: $3 \mathrm{~N}$ ), respectively. The deposition temperature was maintained at $200{ }^{\circ} \mathrm{C}$. The targets were sputtered in defined argon with the pressure of $150 \mathrm{mPa}$. The conditions indicated that deposition rates of Pd and Pt were $0.26 \mathrm{~mm} / \mathrm{s}$ and $0.14 \mathrm{~nm} / \mathrm{s}$, respectively. The Rutherford backscattering spectroscopy (RBS) was performed to determine quantitatively amounts of catalysts on silicon substrates by using a 3.0-MV single-ended accelerator at JAEA/Takasaki. The $2.0 \mathrm{MeV}$ He ions were irradiated with respect to the surface normal of the catalytic layers. The backscattered He ions were detected at an angle of $165^{\circ}$ with respect to the incident direction by using a surface barrier detector. The irradiation was performed with a flux of approximately $8 \times 10^{12}$ ions $/\left(\mathrm{cm}^{2} \times \mathrm{s}\right)$ and a fluence of up to $1.6 \times 10^{15}$ ions $/ \mathrm{cm}^{2}$. Surface morphologies of the samples were observed by using FE-SEM (JSM-6700F, JEOL). Electron beam was operated by a voltage of $10 \mathrm{kV}$ in a vacuum of $1 \times 10^{-4}$

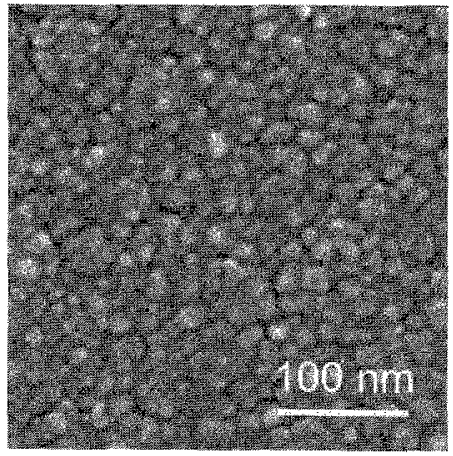

Fig.2. SEM image of amorphous $\mathrm{WO}_{3}$ film.
Pa: To examine the gasochromic properties of the samples, the apparatus was built up as shown in Fig. 1. Change of light transmittance for the samples was measured using a red light emitting diode (LED) during samples were exposed to $1 \%$ hydrogen gas diluted by nitrogen gas $\left(\mathrm{N}_{2}-1 \% \mathrm{H}_{2}\right)$ : The flow rate of the hydrogen gas was controlled at $100 \mathrm{sccm}$ by a mass flow meter.

\section{RESULTS AND DISCUSSIONS}

Figure 2 shows SEM image of an amorphous $\mathrm{WO}_{3}$ film deposited on $\mathrm{SiO}_{2}$ substrate. It is recognized that the film consists of particles with a diameter of approximately $20 \mathrm{~nm}$. The surface roughness of the film is estimated to be $13.0 \mathrm{~nm}$ by using atomic force microscope (AFM). It is difficult to examine practically the surface morphology of catalysts on the $\mathrm{WO}_{3}$ films. Therefore, in this study for investigation of the surface morphology on the catalysts, the Pd and Pt layers were deposited on silicon substrates.

Figure 3 shows RBS spectra of Pd catalysts on the silicon wafers with deposition time of (a) 2, (b) 5, and (c) $20 \mathrm{~s}$. The peaks originating from Pd layers and $\mathrm{Si}$ substrates are recognized at a backscattered energy of $1720 \mathrm{keV}$ and below $1130 \mathrm{keV}$, respectively. When the total yields from the Pd are compared to the yields of $\mathrm{Si}$ in all spectra, amounts of Pd are estimated to be $5.5 \times$ $10^{15}, 1.1 \times 10^{16}$, and $3.6 \times 10^{16}$ atoms $/ \mathrm{cm}^{2}$, in Fig. 3 (a), (b), and (c), respectively. The amounts of the $\mathrm{Pd}$ catalysts can be converted to a thickness of $0.81,1.6$, and $5.3 \mathrm{~nm}$ in Pd layer respectively by using an atomic density of $6.8 \times 10^{22}$ atoms $/ \mathrm{cm}^{3}$ in Pd bulk.

Figure 4 shows the SEM images of the Pd layers on silicon substrates with amounts of (a) $5.5 \times 10^{15}$, (b) 1.1 $\times 10^{16}$, and (c) $3.6 \times 10^{16}$ atoms $/ \mathrm{cm}^{2}$. Pd particles grow on the substrates at the beginning of deposition, by the amounts reach up to $2 \times 10^{16}$ atoms $/ \mathrm{cm}^{2}$. When Pd was deposited to above $3.6 \times 10^{16}$ atoms $/ \mathrm{cm}^{2}$, continuous layer is observed on the surface of silicon substrate. In

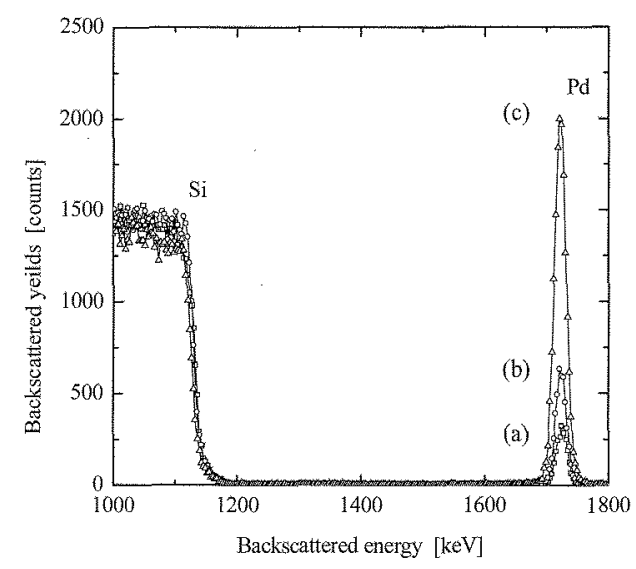

Fig.3. RBS spectra of Pd catalysts on the silicon wafers with a deposition time of (a) 2 , (b) 5 , and (c) $20 \mathrm{~s}$. 

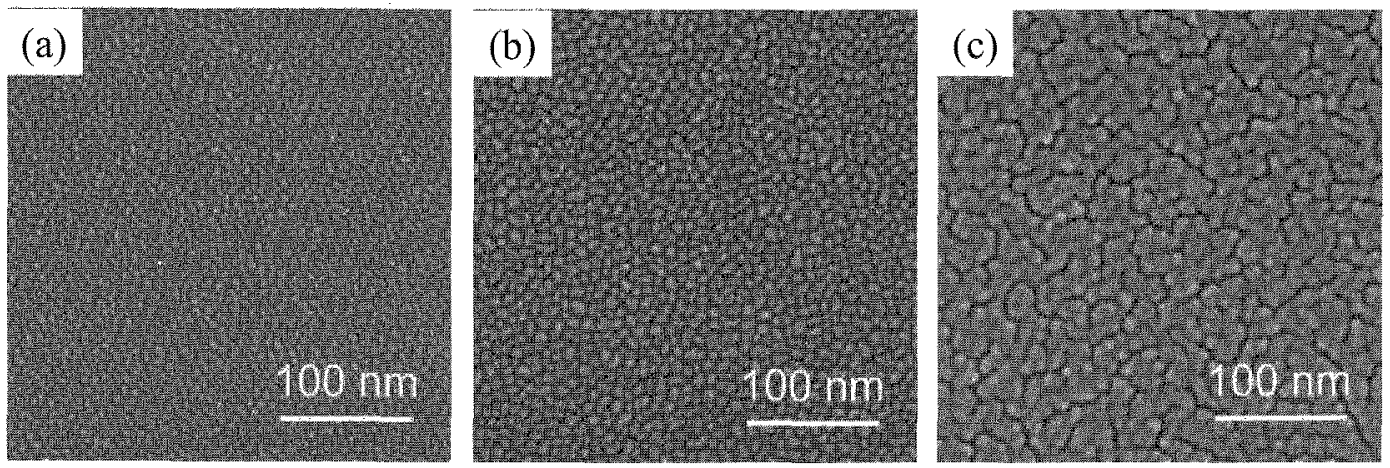

Fig.4. SEM images of Pd catalysts on the silicon substrate with amounts of (a) $5.5 \times 10^{15}$, (b) $1.1 \times 10^{16}$, and (c) $3.6 \times$ $10^{16}$ atoms $/ \mathrm{cm}^{2}$.

also Pt layers, the catalytic particles grew to continuous layers with increasing the amounts of Pt. The critical point of Pt amounts for continuous layers was estimated to be $2 \times 10^{15}$ atoms $/ \mathrm{cm}^{2}$, the critical amounts are one order less than that of Pd layer. The difference of the critical points between $\mathrm{Pd}$ and $\mathrm{Pt}$ layers originates from the particle sizes; Pt particles one order nm smaller than $\mathrm{Pd}$ particles.

Figure 5 shows coloration behavior in $\mathrm{WO}_{3}$ films deposited on $\mathrm{SiO}_{2}$ substrates with $\mathrm{Pd}$ of (a) $5.5 \times 10^{15}$, (b) $1.1 \times 10^{16}$, and (c) $3.6 \times 10^{16}$ atoms $/ \mathrm{cm}^{2}$. The lateral axis in Fig. 5 shows normalized transmittance at a wavelength of $650 \mathrm{~nm}$ for initial transmittance. When $\mathrm{N}_{2}-1 \% \mathrm{H}_{2}$ gas flow, the transmittance drastically degreases with increasing the exposure time and almost saturates above $400 \mathrm{~s}$ in all samples. The transmittances for colored states become lower with increasing the amounts of $\mathrm{Pd}$ catalysts. However, there is no difference in coloration speed at the beginning of hydrogen flowing, unlike previous reports $[4,6]$. It is suggested that gasochromic coloration is limited by hydrogen transfer into $\mathrm{WO}_{3}$ films [4]. In order to examine the effects of catalysts on gasochromic properties by varying the amounts of catalysts, the transmittances for colored

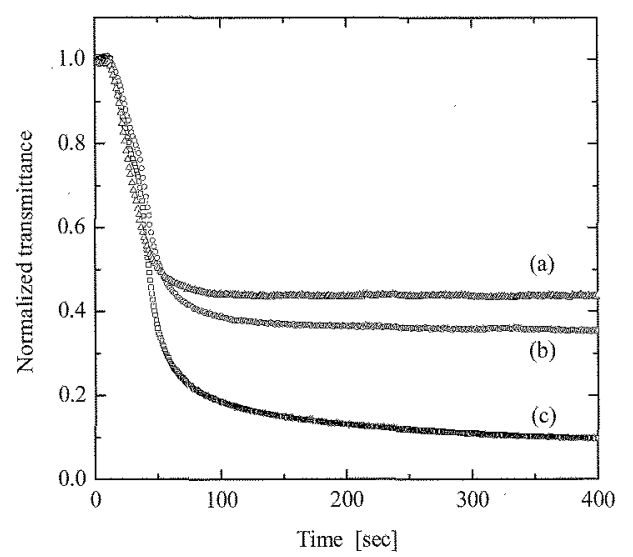

Fig.5. Gasochromic coloration in $\mathrm{WO}_{3}$ films with $\mathrm{Pd}$ catalysts of (a) $5.5 \times 10^{15}$, (b) $1.1 \times 10^{16}$, and (c) $3.6 \times 10^{16}$ atoms $/ \mathrm{cm}^{2}$. states after 20 min passed from hydrogen gas flow were measured. Figure 6 shows normalized transmittance of colored samples with various amounts of $\mathrm{Pd}$ and $\mathrm{Pt}$ catalysts. For Pd deposited films, the coloration levels are improved along with increasing the amounts of $\mathrm{Pd}$ catalysts. For higher amounts of $\mathrm{Pd}$ than $1 \times 10^{16}$ atoms $/ \mathrm{cm}^{2}$, the coloration levels reach to constant values. The constant coloration levels with great amounts catalysts are considered to originate from saturation of hydrogen incorporation into the $\mathrm{WO}_{3}$ film. The amounts of $1 \times 10^{16}$ atoms $/ \mathrm{cm}^{2}$ of $\mathrm{Pd}$ catalysts correspond to the layer thickness enough to cover throughout the $\mathrm{WO}_{3}$ surface, referring the SEM observation. On the other hand, excellent properties are obtained with greater amounts of Pt catalysts than $2 \times 10^{15}$ atoms $/ \mathrm{cm}^{2}$, the amounts also correspond to cover the whole surface of the $\mathrm{WO}_{3}$ films. The results suggest that excellent gasochromic properties require to the catalytic layers with covering the whole $\mathrm{WO}_{3}$ surfaces. It is implied that gasochromic coloration was limited by migration of hydrogen atoms between the catalytic layers and the $\mathrm{WO}_{3}$ films, supported by no difference of coloration speed with various catalytic layer thicknesses in Fig. 5. In the optical hydrogen sensors using gasochromic $\mathrm{WO}_{3}$

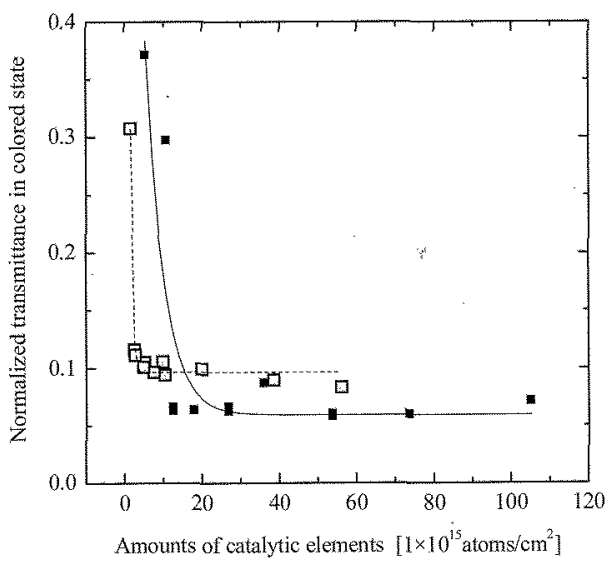

Fig.6. Gasochromic coloration levels of $\mathrm{WO}_{3}$ films with varying $\mathrm{Pd}$ (solid squire) and $\mathrm{Pt}$ (blank squire) catalysts. 
films, Pt catalysts are more suitable than Pd catalysts. It is found that controlling the particle size of catalysts leads to improvement of sensitivity in the optical hydrogen sensors using gasochromic $\mathrm{WO}_{3}$ films.

\section{CONCLUSION}

Effects of $\mathrm{Pd}$ and $\mathrm{Pt}$ catalyst on gasochromic properties in amorphous $\mathrm{WO}_{3}$ films were investigated. The particles of $\mathrm{Pd}$ and $\mathrm{Pt}$ grew on the surface at beginning of deposition and the continuous catalytic layers were observed with increasing the amounts of catalysts. The critical points can be evaluated to be $1 \times$ $10^{16}$ and $2 \times 10^{15}$ atoms $/ \mathrm{cm}^{2}$ of Pd and Pt catalysts, respectively. The $\mathrm{WO}_{3}$ films with continuous catalytic layers on $\mathrm{SiO}_{2}$ substrates showed excellent gasochromic properties. The results indicate that Pt catalysts are suitable compared to Pd catalysts in hydrogen sensors using gasochromic $\mathrm{WO}_{3}$ films. It was found that controlling the catalysts into small catalytic particles and continuous layer structure makes a possible to improve the sensitivities of the optical hydrogen sensors.

\section{ACKNOWREDGMENTS}

This work was supported by a Grant-in-Aid for Scientific Research(C) from the Japan Society for the Promotion of Science (No. 19560717).

\section{REFERENCE}

[1] C. Christofides, A. Mandelis, J. Appl. Phys. 68 R1-R30 (1990).

[2] K. Itoh, T. Ohgami, Appl. Phys. Lett. 60 938-940 (1992).

[3] S. H. Lee, H. M. Cheong, P. Liu, D. Smith, C. E. Tracy, A. Mascarenhas, J. R. Pitts, S. K. Deb, Electrochimica Acta 46 1995-1999 (2001).

[4] D. Schweiger, A. Georg, W. Graf, V. Wittwer, Solar Energy and Solar Cells 54 99-108 (1998).

[5] H. Shanak, H. Schmitt, J. Nowoczin, C. Ziebert, Solid State Ionics 171 99-106(2004).

[6] A. Georg, W. Graf, D. Schweiger, V. Wittwer, P. Nitz, H. R. Wilson, Solar Energy 62 215-228 (1998).

[7] H. Nakagawa, N. Yamamoto, S. Okazaki, T. Chinzei, S. Asakura, Sens. Actuators B 93 468-474 (2003).

[8] A. Inouye, S. Yamamoto, S. Nagata, K. Takano, M. Yoshikawa, T. Shikama, Trans. Mater. Res. Soc. Jpn. 32 107-110 (2007).

(Recieved December 9, 2007 ; Accepted September 1; 2008) 